\title{
LIVER
}

\section{Age and paracetamol self-poisoning}

\section{E Schmidt}

Gut 2005;54:686-690. doi: 10.1136/gut.2004.054619

Background: Whereas paracetamol poisoning is predominantly seen in adolescents and young adults, the majority of paracetamol associated deaths occur in an older population.

Aims: The aim of the present study was to evaluate age as a risk factor for fulminant hepatic failure (FHF) and death in a large population of patients with paracetamol poisoning.

Patients: A total of 746 patients transferred to a specialised unit with severe paracetamol poisoning and 273 unselected patients admitted from the local region over a 10 year period.

Methods: A partly retrospective study based on hospital charts. The risk associated with age was evaluated by multivariate analysis.

Results: Paracetamol poisoning most frequently occurred in the age group 15-24 years. Transferred

Correspondence to: Dr LE Schmidt,

Department of 'Hepatology A. 2.12.1, Rigshospitalet, Blegdamsvej 9, DK-2100 Copenhagen $\varnothing$, Denmark; lars.schmidt@dadlnet.dk

Revised version received 8 January 2005 Accepted for publication 18 January 2005 patients were significantly older than local patients (median age 37 years $v 29$ years; $p=0.0006$ ). In contrast, FHF and death from paracetamol poisoning most frequently occurred in patients aged 40 years or above. In a logistic regression analysis, "age $\geqslant 40$ years" was associated with an excess risk of FHF (odds ratio (OR) 2.33 (95\% confidence interval (CI) 1.50-3.64)) and death or liver transplantation (OR 4.18 (95\% Cl 2.17-8.05)). In addition, older age was associated with other risk factors for paracetamol hepatotoxicity such as regular alcohol abuse and late presentation.

Conclusions: Age 40 years or above was identified as a significant independent risk factor for FHF and mortality following paracetamol overdose. Patients aged 40 years or above should be considered as high risk patients, in particular when older age appears in combination with regular alcohol abuse or late presentation.
$\mathrm{P}$ aracetamol (acetaminophen) poisoning is an increasing health problem, and paracetamol induced hepatotoxicity has become the leading cause of fulminant hepatic failure (FHF) in the Western world..$^{1-3}$ Paracetamol poisoning is predominantly seen in adolescents and young adults. ${ }^{4-8}$ In contrast, the majority of paracetamol associated deaths occur in an older population. ${ }^{79}$

There may be multiple reasons why the outcome of paracetamol poisoning may tend to be more severe in older than in younger individuals. In general, the rate of fatal to non-fatal self-harm increases with age. ${ }^{10}{ }^{11}$ This may be explained by a higher degree of suicidal intent in the elderly, which again may reflect in more severe self-poisoning (for example, by ingestion of a larger overdose). ${ }^{5}$ 12 In addition, late life suicidal activity is frequently associated with alcohol abuse, ${ }^{13}$ which is a known risk factor of paracetamol induced hepatotoxicity. ${ }^{14}$ Furthermore, age related changes in functioning liver cell mass or in the susceptibility to paracetamol may increase the risk of developing FHF following a paracetamol overdose. ${ }^{15} 16$

Several studies have identified older age (above 40 or 50 years) as a risk factor of mortality from non-paracetamol FHF whereas age has not conclusively been associated with survival from paracetamol induced FHF. ${ }^{17-19}$ However, if the chance of survival from paracetamol induced FHF decreases with age, this would further contribute to the overall higher mortality from paracetamol poisoning in the older population.

The aim of this study was to evaluate age as a risk factor for FHF and death from paracetamol induced hepatotoxicity in a large population of patients with paracetamol poisoning.

\section{PATIENTS AND METHODS}

The charts of all patients admitted to Rigshospitalet, Copenhagen, Denmark, with paracetamol poisoning between 1994 and 2003 were reviewed. The following information was recorded for each case: age, sex, whether the patient was transferred from another hospital, cause of the self-poisoning, quantity of paracetamol ingested, time from paracetamol ingestion to presentation at hospital, time from paracetamol ingestion to $\mathrm{N}$-acetylcysteine (NAC) treatment ("time to NAC"), regular alcohol abuse, acute co-ingestion of alcohol, regular medication, concomitant drug overdosing, peak values of the international normalised ratio (INR), alanine transaminase (ALT), and creatinine, hepatic encephalopathy (HE), orthotopic liver transplantation (OLT) performed, and death/survival. Regular abuse of alcohol was defined as an excess of 14 units weekly for women and 21 units for men ( 1 unit equalling $10 \mathrm{~g}$ of ethanol). HE was graded according to the Fogarty criteria, and FHF was defined as the development of HE grade II-IV. ${ }^{20}$

In accordance with the Danish recommendations, all patients with diagnosed or suspected paracetamol poisoning were immediately treated with a standard regimen of intravenous NAC for a minimum of 20 hours, regardless of time to presentation or risk assessment. ${ }^{21}$ The Department of Hepatology at Copenhagen University Hospital is the tertiary care centre of liver disease in Denmark, and the majority of patients are transferred from other Danish hospitals. Indication for considering transfer of a patient to the tertiary care centre are: INR $>1.7$, creatinine $>300 \mu \mathrm{mol} / \mathrm{l}$, platelet count $<50 \times 10^{9} / 1$, arterial $\mathrm{pH}<7.30$ after fluid resuscitation, or the presence of HE. ${ }^{21}$ However, the hospital also serves as a primary hospital for the local region in the Copenhagen area. Thus some patients are primarily admitted from the local region without any selection, typically via the hospital's emergency room. All patients were treated and monitored in

Abbreviations: ALT, alanine transaminase; FHF, fulminant hepatic failure; $\mathrm{HE}$, hepatic encephalopathy; INR, international normalised ratio; NAC, N-acetylcysteine; OLT, orthotopic liver transplantation 

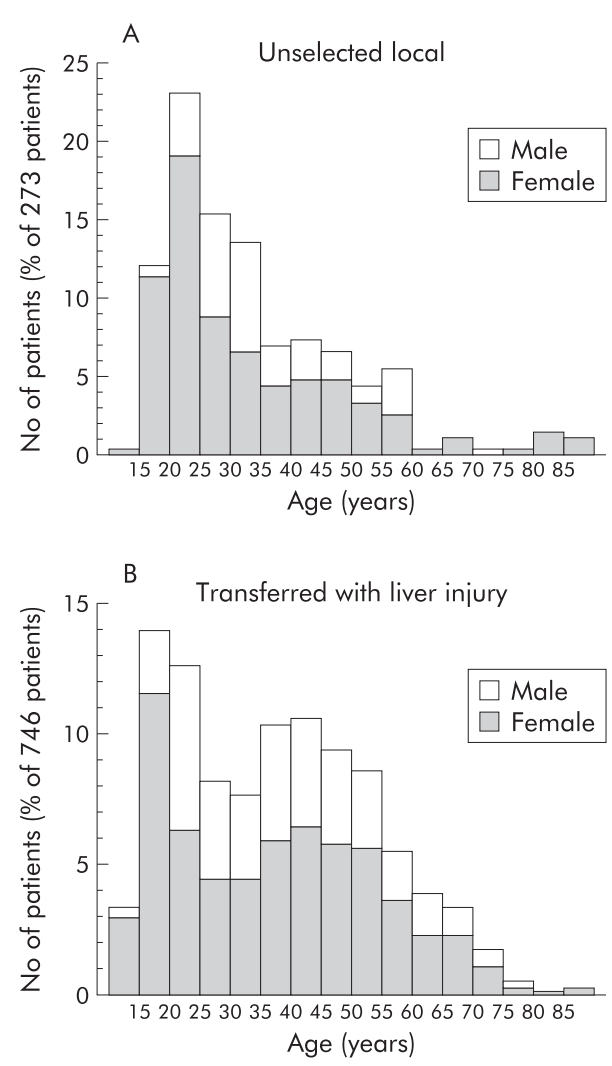

Figure 1 Age and sex specific distributions normalised as a percentage among 273 unselected patients (A) admitted from the local region with predominantly uncomplicated paracetamol poisoning and among 746 patients transferred $(B)$ with signs of paracetamol induced liver injury.

accordance with department standards. Patients fulfilling the King's College Hospital criteria were considered for OLT. ${ }^{18}$

\section{Statistics}

The Mann-Whitney test was used for comparison of a variable between two subgroups and the $\chi^{2}$ test was used for comparison of frequencies. For multivariate analyses, a backward stepwise logistic regression analysis was applied. Data were analysed using the SAS 8.02 statistical software (SAS Institute Inc., Cary, North Carolina, USA). A p value $<0.05$ was considered statistically significant.

\section{RESULTS}

During the 10 year study period, a total of 1019 patients were admitted with paracetamol overdose. The majority of patients $(746(73 \%))$ were transferred from other hospitals whereas the remaining $273(27 \%)$ patients were admitted directly from the local region. Of 746 transferred patients, $170(23 \%)$ developed FHF, 72 (9.7\%) died without OLT, and 11 (1.5\%) were transplanted. In comparison, only two of 273 unselected local patients $(0.7 \%)$ developed FHF and both survived without OLT.

The age and sex specific distributions of the local versus the transferred patients are illustrated in fig l. Local patients showed a positively skewed distribution (fig 1A) with a maximum for the 20-24 year age group (median 29 years; interquartile range 22-41; range 13-89). In comparison, transferred patients had a bimodal distribution (fig 1B) with an absolute maximum at approximately 20 years and a secondary maximum for the 40-44 year age group (median 37 years; interquartile range 22-49; range 12-86). Overall,

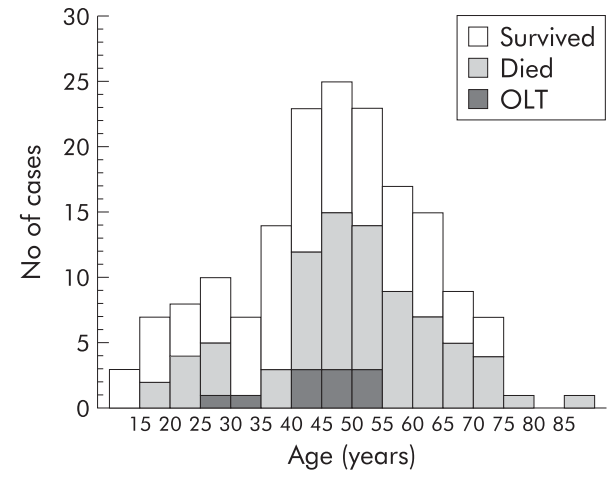

Figure 2 Age distribution among 170 transferred patients with paracetamol induced liver failure with indications of death and orthotopic liver transplantation (OLT).
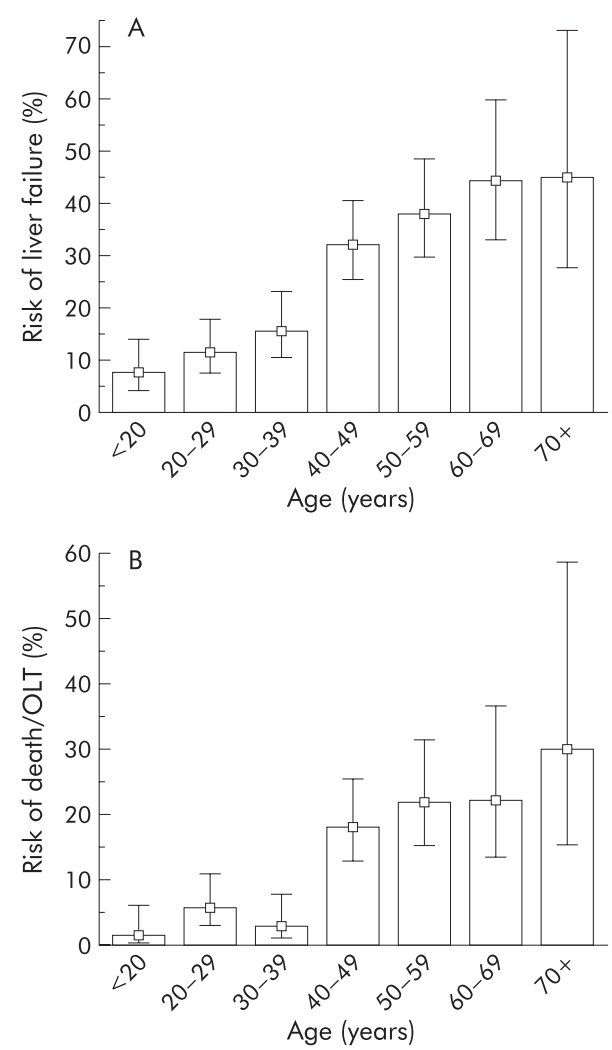

Figure 3 Age specific risk of liver failure (A) and death or orthotopic liver transplantation (OLT) (B) among 746 patients transferred with signs of severe paracetamol intoxication. Whiskers indicate $95 \%$ confidence interval.

transferred patients were significantly older than local patients (Mann-Whitney, $\mathrm{p}=0.0006$ ).

In contrast with the above findings, 170 transferred patients who developed FHF had an almost symmetrical age distribution (fig 2) with a maximum for the 45-49 year age group (median 48 years; interquartile range 38-56; range 12-86). Among transferred patients, those with FHF were significantly older than those without FHF (median $48 v$ 33 years; Mann-Whitney, $\mathrm{p}<0.00001)$. Age distribution among 83 patients who either died or required OLT was very similar (fig 2), with a maximum for the $45-49$ year age group (median 49 years; interquartile range 41-58; range 18-86). 
Table 1 Comparison of anamnestic, biochemical, and clinical data for 746 transferred patients, divided on the basis of age less than or greater than 40 years

\begin{tabular}{lcc} 
& $\begin{array}{c}\text { Age }<\mathbf{4 0} \text { years } \\
\text { (n=418) }\end{array}$ & $\begin{array}{c}\text { Age } \geqslant 40 \text { years } \\
(\mathbf{n = 3 2 8})\end{array}$ \\
\hline Sex (M/F) & $153(37 \%) / 265(63 \%)$ & $121(37 \%) / 207(63 \%)$ \\
Quantity of paracetamol (mg/kg) & $448(329-694)$ & $500(311-791)$ \\
Time to presentation at hospital (h) & $19(12-36)$ & $24(15-48)^{* * *}$ \\
Time to N-acetylcysteine (h) & $20(12-38)$ & $30(17-49)^{* * *}$ \\
Acute alcohol co-ingestion & $77(18 \%)$ & $52(16 \%)$ \\
Regular alcohol abuse & $57(14 \%)$ & $161(49 \%)^{* * *}$ \\
Acetylsalicylic acid co-overdose & $13(3.1 \%)$ & $16(4.9 \%)$ \\
Benzodiazepine co-overdose & $43(10 \%)$ & $71(22 \%)^{*}$ \\
Opioid co-overdose & $18(3.4 \%)$ & $23(7.0 \%)$ \\
Accidental paracetamol overdose & $47(11 \%)$ & $88(27 \%)^{*}$ \\
International normalised ratio & $2.9(2.2-4.4)$ & $3.6(2.6-5.5)^{* * *}$ \\
Alanine transaminase (U/l) & $8166(2400-11340)$ & $6388(3089-9560)$ \\
Creatinine ( $\mu$ mol/l) & $90(76-150)$ & $160(87-366)^{* * *}$ \\
Liver failure & $49(12 \%)$ & $121(37 \%)^{* *}$ \\
Liver transplantation & $2(0.5 \%)$ & $9(2.7 \%)^{*}$ \\
Death & $13(3.1 \%)$ & $59(18 \%)^{* * *}$ \\
\hline Values are given as median (1 st quartile-3rd quartile). & \\
*p<0.05, ***p<0.00001 ( $\chi^{2}$ or Mann-Whitney's test). & \\
\hline
\end{tabular}

However, among transferred patients with FHF, patients who died or required OLT were significantly older than those who survived without OLT (Mann-Whitney, $\mathrm{p}=0.02$ ). Ten of 11 patients transplanted survived to discharge and the only fatality was in a woman aged 41 years.

Figure 3 illustrates the risk of developing FHF and risk of death or OLT for the different age groups of transferred patients. In a pairwise comparison, the risk for each of the age groups above 40 years was significantly higher than that of the age groups below 40 years. In contrast, no significant differences were demonstrated between any two age groups below 40 years or between any two age groups above 40 years. Consequently, a 40 year age threshold was found to discriminate between a high risk and a low risk group. Thus age $\geqslant 40$ years was associated with a significantly increased risk of FHF (risk ratio 3.15 (95\% confidence interval (CI) 2.33-4.24)) as well as death or OLT (risk ratio 5.78 (95\% CI 3.37-9.91)). Moreover, mortality in patients with FHF was also significantly increased in those aged 40 years or above (risk ratio 1.84; 95\% CI 1.17-2.88)).

In addition to being associated with a poorer outcome, age $\geqslant 40$ years was also significantly associated with a longer "time to NAC", a higher prevalence of regular alcohol abuse, and a higher incidence of benzodiazepine co-overdose

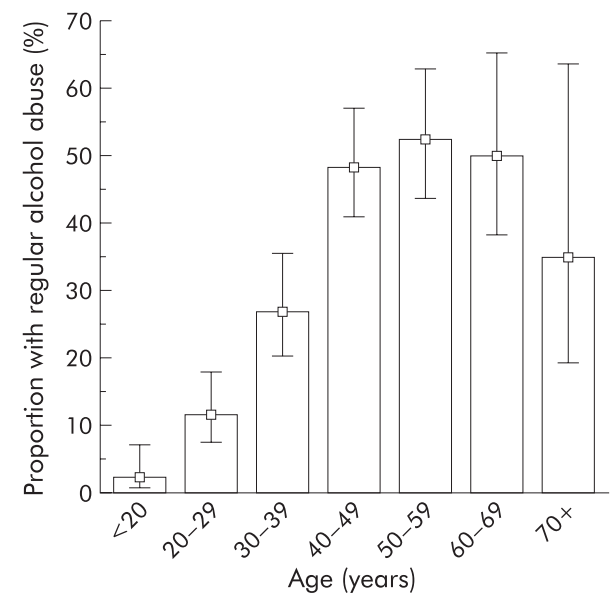

Figure 4 Age specific prevalence of regular alcohol abuse among 746 patients transferred with signs of severe paracetamol intoxication. Whiskers indicate $95 \%$ confidence interval. (table 1). In particular "time to NAC" and regular alcohol abuse have been identified as risk factors of paracetamol induced hepatotoxicity. ${ }^{14}$ As illustrated in fig 4, the prevalence of regular alcohol abuse was significantly higher in those age groups that had the worst outcome. Similarly, "time to NAC" was shown to increase with age (fig 5). The main reason for the delayed onset of NAC in older patients was that they presented later (table 2), which may be explained in part by the higher proportion of accidental overdose in older patients, as accidental versus suicidal overdose was strongly associated with late presentation (median 42 v 20 hours; Mann-Whitney, $\mathrm{p}<0.00001$ ). In 93\% of cases, the diagnosis of paracetamol poisoning was made at the initial contact with the hospital, and NAC was initiated without delay. An "inhospital" delay due to late diagnosis was more common in patients aged 40 years or more compared with those $<40$ years (delay in $11 \% \vee 3.4 \%$; $\chi^{2}$ test, $\left.\mathrm{p}=0.0001\right)$. However, this delay had only a minor contribution (average two hours per patient) to the overall difference in "time to NAC" between the groups.

In order to establish the independent risk associated with age, a multivariate analysis using "age $\geqslant 40$ years" together with known or suspected risk factors of paracetamol induced hepatotoxicity (sex, weight, quantity of paracetamol, "time to

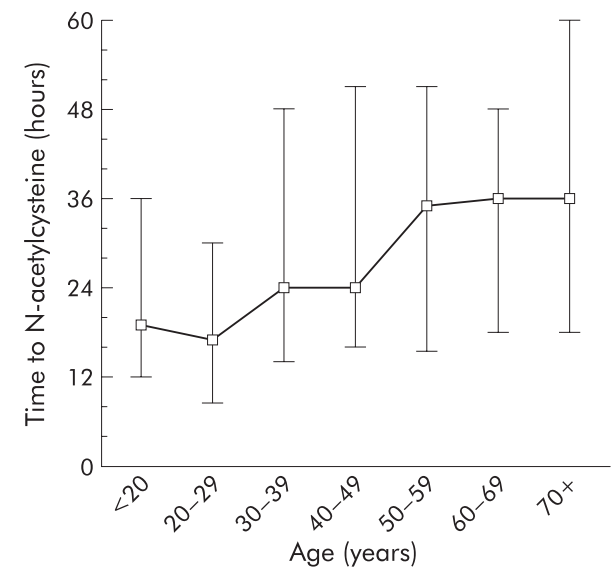

Figure 5 Age specific distribution of time from paracetamol ingestion to initiation of $\mathrm{N}$-acetylcysteine therapy in 746 patients transferred with signs of severe paracetamol intoxication. Plot indicates median and interquartile range (whiskers). 
Table 2 Results of logistic regression analysis presenting identified risk factors of fulminant hepatic failure and mortality (including liver transplantation) in order of statistical significance in 746 transferred patients

\begin{tabular}{lll}
\hline Risk factor & Odds ratio & p Value \\
\hline Fulminant hepatic failure & & \\
Time to N-acetylcysteine & $1.36(1.23-1.50)$ per $12 \mathrm{~h}$ & $<0.00001$ \\
Regular alcohol abuse & $2.92(1.88-4.53)$ & $<0.00001$ \\
Age $\geqslant 40$ years & $2.33(1.50-3.64)$ & 0.0002 \\
Acute alcohol ingestion & $0.39(0.20-0.74)$ & 0.004 \\
Benzodiazepine co-overdose & $1.75(1.05-2.93)$ & 0.03 \\
Mortality & $3.72(2.07-6.67)$ & 0.00001 \\
Regular alcohol abuse & $4.18(2.17-8.05)$ & 0.00002 \\
Age $\geqslant 40$ years & $1.25(1.11-1.40)$ per $12 \mathrm{~h}$ & 0.0001 \\
Time to N-acetylcysteine & $0.13(0.04-0.46)$ & 0.001 \\
Acute alcohol ingestion & $3.78(1.25-11.5)$ & 0.02 \\
Acetylsalicylic acid co-overdose & $1.95(1.07-3.56)$ & 0.03 \\
Female sex & & \\
\hline Odds ratios are given with 95\% confidence interval. &
\end{tabular}

NAC", regular alcohol abuse, acute alcohol co-ingestion, acetylsalicylic acid co-overdose, benzodiazepine co-overdose, and opioid co-overdose) as independent variables was performed. ${ }^{14} 22$ In this logistic regression analysis, the three major risk factors were "time to NAC", regular alcohol abuse, and "age $\geqslant 40$ years" (table 2 ). Thus "age $\geqslant 40$ years" was an independent risk factor of FHF (odds ratio 2.33 (95\% CI 1.503.64)) and of death or OLT (odds ratio 4.18 (95\% CI 2.17$8.05)$ ). The independent contribution of "age $\geqslant 40$ years" to the risk of FHF and mortality is illustrated in fig 6, in which patients were divided into four subgroups by regular alcohol
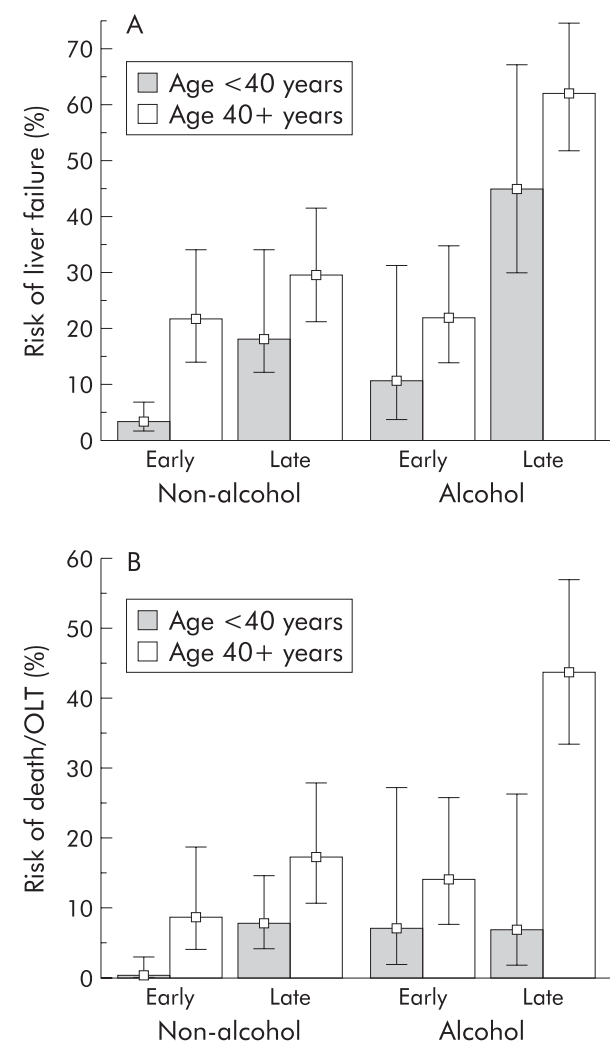

Figure 6 Age specific risk of liver failure (A) and death or liver transplantation (OLT) (B) among 746 patients transferred with signs of severe paracetamol intoxication divided into four subgroups by regular alcohol abuse (absent or present) and time of $\mathrm{N}$-acetylcysteine treatment (early (that is, $<24$ hours) or late (that is, $>24$ hours)). Whiskers indicate $95 \%$ confidence interval. abuse (absent or present) and "time to NAC" (early (that is, $<24$ hours) or late (that is, $>24$ hours)). For every combination of regular alcohol abuse and "time to NAC", an excess risk was observed in those aged $\geqslant 40$ years.

\section{DISCUSSION}

In the present study, age over 40 years was independently associated with an increased risk of development of FHF and death or OLT following paracetamol overdose. In addition, older age was associated with other risk factors, such as alcohol abuse and late presentation, which further contributed to a poorer prognosis in this age group.

Paracetamol is the drug most commonly used in deliberate drug overdoses in adolescents, and many studies have specifically focused on this age group. ${ }^{62324}$ Indeed, paracetamol overdose is most common in younger people and in first time self-overdose cases, whereas overdose with sedatives and antidepressants is preferred by the elderly and in repeated self-overdose. ${ }^{5}{ }^{9}$ This pattern may well reflect differences in availability, as most adolescents have easy access to paracetamol..$^{25}$ In the present study, the age specific distribution among the unselected population from the local region showed the expected distribution, with a predominance of cases in adolescents and young adults. In comparison, transferred patients were significantly older. This may reflect the fact that the region of Copenhagen is not representative of the entire country but most likely it results from selection. Thus it may be assumed that a greater proportion of older rather than younger individuals fulfil the Danish criteria for transfer to the tertiary liver centre. Transferred patients represent the 5\% most severe cases of paracetamol poisoning, including most cases of paracetamol induced FHF in Denmark. This would imply an underlying rate of paracetamol induced FHF of approximately $1 \%$ in the external referral base, which corresponds to the finding of a $0.7 \%$ rate of FHF from the local region. Similarly, the fatality rate in the external referral base may be estimated at $0.5 \%$, which is comparable with rates previously reported from the UK $(0.4 \%),{ }^{26}$ France $(0.1 \%)$, Scotland $(1.6 \%),{ }^{9}$ and the USA $(0.8 \%) .^{27}$

In contrast with the above findings, the majority of paracetamol deaths in the present study occurred in older adults, and deaths among adolescents were rare. This is in agreement with recent findings from the Scottish poison registry. ${ }^{9}$ As the same transfer criteria were applied in all age groups in the present study, the difference in mortality rate between age groups may not be explained by differences in the severity of hepatotoxicity. The study revealed several explanations for the poorer prognosis in older adults. Most importantly, multivariate analysis identified older age as an 
independent risk factor of FHF and death or OLT from paracetamol overdose. In addition, older age was associated with known risk factors of paracetamol hepatotoxicity such as regular alcohol abuse and late presentation. ${ }^{14}$ The incidence of chronic alcohol abuse was very high in the 4069 year age group whereas it was rare in young adults. Similarly, late presentation was particularly frequent in the age group $>50$ years. Consequently, a high risk subgroup of patients characterised by the not uncommon triad of older age, alcohol abuse, and late presentation was identified. In contrast, the typical paracetamol overdose case, a young person without alcohol abuse, who presents early after paracetamol ingestion, could be demonstrated to have an excellent prognosis. Also, accidental (versus suicidal) paracetamol overdose, which has been associated with a poor prognosis, was more common in older than in younger adults. $^{28}$ However, the apparent excess risk of accidental overdose is probably largely explained by the association with late presentation and alcohol abuse. ${ }^{14}$

Although the present study identified age as a significant independent risk factor of paracetamol hepatotoxicity, the underlying mechanisms by which age increases the risk remain unclear, but are most likely multiple. Firstly, older individuals were more likely than younger individuals to develop FHF from paracetamol overdose. There is no evidence to suggest that paracetamol metabolism dramatically differs with age. ${ }^{15}$ Possibly, younger individuals may simply tolerate more extensive liver injury due to having a larger functioning liver cell mass prior to the overdose. ${ }^{16}$ This mechanism may be supported by the observation from the present study that the extent of hepatic injury, estimated by peak ALT, actually tended to be higher $(p=0.07)$ in younger than in older individuals whereas hepatic dysfunction estimated by INR was significantly more pronounced in the older age group. Alternatively, younger individuals may better compensate for acute hepatic dysfunction (for example, via a higher capacity for extrahepatic metabolism of toxic substances such as ammonia which may be substantially metabolised by striated muscle tissue). ${ }^{29}$ Secondly, mortality from developed FHF was higher in older than in younger individuals. Age has previously been identified as a risk factor for mortality from non-paracetamol FHF such as fulminant hepatitis B. ${ }^{17-19} \mathrm{~A}$ trend has also been suggested towards a higher mortality with increasing age from paracetamol induced FHF. ${ }^{18}$ Possibly, younger individuals are less susceptible to potentially fatal complications of FHF such as infection and cardiovascular dysfunction. ${ }^{30}$ Alternatively, the capacity for hepatic regeneration may be impaired in older individuals leading to a reduced chance of recovery.

In conclusion, age $\geqslant 40$ years was identified as a significant independent risk factor of FHF and mortality following paracetamol overdose. Thus older age was not only associated with a high risk of developing FHF, but also with a high mortality from developed FHF. Patients aged 40 years or more should be considered as high risk patients, in particular when older age appears in combination with regular alcohol abuse or late presentation.

Conflict of interest: None declared.

\section{REFERENCES}

1 Larsen FS, Kirkegaard P, Rasmussen A, et al. The Danish liver transplantation program and patients with serious acetaminophen intoxication. Transplant Proc 1995;27:3519-20.

2 Makin AJ, Wendon J, Williams R. A 7-year experience of severe acetaminophen-induced hepatotoxicity. Gastroenterology 1995; 109:1907-16

3 Ostapowicz G, Fontana RJ, Schiødt FV, et al. Results of a prospective study of acute liver failure at 17 tertiary care centers in the United States. Ann Intern Med 2002; 137:947-54

4 Peterson RG, Rumack BH. Age as a variable in acetaminophen overdose. Arch Intern Med 1981;141:390-3.

5 Wynne $\mathbf{H}$, Bateman DN, Hassanyeh F, et al. Age and self-poisoning: the epidemiology in Newcastle upon Tyne in the 1980s. Hum Toxicol 1987;6:511-15.

6 Townsend E, Hawton K, Harriss L, et al. Substances used in deliberate selfpoisoning 1985-1997: trends and associations with age, gender, repetition and suicide intent. Soc Psychiatry Psychiatr Epidemiol 2001;36:228-34.

7 Schmidt LE. Paracetamol self-poisoning among adolescents in a department of hepatology. Int J Adolesc Med Health 2001;13:327-34.

8 Hawton K, Fagg J, Simkin S, et al. Deliberate self-harm in adolescents in Oxford, 1985-1995. J Adolesc 2000;23:47-55.

9 Bateman DN, Bain M, Gorman D, et al. Changes in paracetamol, antidepressants and opioid poisoning in Scotland during the 1990s. QJM 2003;96:125-32

10 De Leo D, Padoani W, Scocco P, et al. Attempted and completed suicide in older subjects: results from the WHO/EURO multicentre study of suicidal behaviour. Int J Geriatr Psychiatry 2001;16:300-10.

11 Spicer RS, Miller TR. Suicide acts in 8 states: incidence and case fatality rates by demographics and method. Am J Public Health 2000;90:1885-91.

12 Lawrence D, Almeida OP, Hulse GK, et al. Suicide and attempted suicide among older adults in Western Australia. Psychol Med 2000;30:813-21.

13 Kennedy GJ, Tanenbaum S. Suicide and aging: international perspectives. Psychiatr Q 2000;71:345-62.

14 Schmidt LE, Dalhoff K, Poulsen HE. Acute versus chronic alcohol consumption in acetaminophen-induced hepatotoxicity. Hepatology 2002;35:876-82.

15 Miller RP, Roberts RJ, Fischer L. Acetaminophen elimination kinetics in neonates, children, and adults. Clin Pharmacol Ther 1976;19:284-94.

16 Marchesini G, Bua V, Brunori A, et al. Galactose elimination capacity and liver volume in aging man. Hepatology 1988;8:1079-83.

17 Bernuau J, Goudeau A, Poynard T, et al. Multivariate analysis of prognostic factors in fulminant hepatitis B. Hepatology 1986;6:648-51.

18 O'Grady JG, Alexander GJ, Hayllar KM, et al. Early indicators of prognosis in fulminant hepatic failure. Gastroenterology 1989;97:439-45.

19 Dhiman RK, Seth AK, Jain S, et al. Prognostic evaluation of early indicators in fulminant hepatic failure by multivariate analysis. Dig Dis Sci 1998;43:1311-16.

20 Leevy CM, Sherlock S, Tygstrup N, et al. Diseases of the liver and the biliary tract. New York: Raven Press, 1994:6-7.

21 Clemmesen JO, OH P, Dalhoff KP, et al. Rekommandation for behandling af paracetamolforgiftning. Ugeskr Læger 1996;158:6892-5.

22 Schmidt LE, Dalhoff K. Concomitant overdosing of other drugs in patients with paracetamol poisoning. Br J Clin Pharmacol 2002;53:535-41.

23 Hawton K, Fagg J, Simkin S, et al. Trends in deliberate self-harm in Oxford, 1985-1995. Implications for clinical services and the prevention of suicide. Br J Psychiatry 1997; 171:556-60.

24 Hawton K, Ware C, Mistry H, et al. Paracetamol self-poisoning. Characteristics, prevention and harm reduction. Br J Psychiatry $1996 ; 168: 43-8$.

25 Gilbertson RJ, Harris E, Pandey SK, et al. Paracetamol use, availability, and knowledge of toxicity among British and American adolescents. Arch Dis Child 1996;75:194-8.

26 Gunnell D, Hawton K, Murray V, et al. Use of paracetamol for suicide and non-fatal poisoning in the UK and France: are restrictions on availability justified? J Epidemiol Community Health 1997;51:175-9.

27 Lee WM. Acetaminophen and the U.S. Acute Liver Failure Study Group: lowering the risks of hepatic failure. Hepatology 2004;40:6-9.

28 Gyamlani GG, Parikh CR. Acetaminophen toxicity: suicidal vs. accidental. Crit Care 2002;6:155-9.

29 Clemmesen JO, Kondrup J, OH P. Splanchnic and leg exchange of amino acids and ammonia in acute liver failure. Gastroenterology 2000;118:1131-9.

30 Ellis A, Wendon J. Circulatory, respiratory, cerebral, and renal derangements in acute liver failure: pathophysiology and management. Semin Liver Dis 1996; 16:379-88 